\title{
ANÁLISE DA UTILIDADE DA PESQUISA DE ASPIRAÇ̄̃O PULMONAR NO EXAME DE CINTILOGRAFIA PARA A AVALIAÇÄO DE REFLUXO GASTROESOFÁGICO
}

Ariadne Gouvea, Michele Santi Costa, flávia Costa Aldighieri, Marco Antônio Condé de Oliveira, Júlio Cesar Rodrigues Pereira, Paulo Schiavom Duarte*

Trabalho realizado na Seção de Medicina Nuclear, Fleury - Centro de Medicina Diagnóstica, São Paulo, SP, Brasil

\section{*Correspondência}

Seção de Medicina Nuclear Rua Cincinato Braga, 232, Paraíso

São Paulo, SP, Brasil, CEP: $01333-910$. psduarte@hotmail.com Tel: (11) 5014-6789 Fax: (11) 5014-6788

\section{RESUMO}

OвıEтıvo. Avaliar a utilidade da realização rotineira da imagem tardia de campos pulmonares cinco horas após a ingestão do radiofármaco, no exame de cintilografia para pesquisa de refluxo gastroesofágico (PRGE), com a finalidade de detectar a presença de aspiração pulmonar.

MéтоDos. Foram analisados retrospectivamente 755 exames (400 homens, 355 mulheres) com idade média de 5, 18 anos (variando de I mês até 94,5 anos). Os exames foram analisados segundo a presença de refluxo, sua freqüência, sua intensidade e a altura do esôfago atingida. Avaliou-se também a presença de atividade radioativa em campos pulmonares nas imagens tardias.

Resultados. Trezentos e sete exames apresentaram episódios de refluxo gastroesofágico: 84 exames apresentaram um episódio, 51 apresentaram dois, 45 apresentaram três, 37 apresentaram quatro, 18 apresentaram cinco e 72 apresentaram mais do que cinco episódios. Em 179 exames observou-se refluxo de grau discreto, 69 de grau moderado, 37 de grau acentuado e em 22 exames o grau de refluxo não foi registrado. Quarenta e seis exames apresentaram refluxo até o terço inferior do esôfago, 83 até o terço médio, 161 até o terço superior, II até a boca e seis exames não tiveram a altura do refluxo registrada. A imagem tardia foi realizada em 753 exames não se observando nenhum episódio de aspiração pulmonar.

Conclusão. A imagem tardia de campos pulmonares na PRGE nunca foi positiva neste grupo de exames, o que pode ser decorrente da raridade de aspiração pulmonar na nossa amostra de pacientes, da baixa sensibilidade do método para detectar aspiração pulmonar ou de ambos os fatores.

Unitermos: Cintilografia. Refluxo. Gastroesofágico. Aspiração. Pulmão.

\section{INTRODUÇÃO}

A cintilografia para a pesquisa de refluxo gastroesofágico tem sido descrita como uma técnica sensível, não-invasiva e fisiológica para a detecção desta patologial-2. Apesar de sua sensibilidade ser limitada quando em comparação à pHmetria ${ }^{3-4}$, ela possui a vantagem de ser mais tolerável pelo paciente. Além disso, alguns trabalhos mostram que estas duas técnicas são complementares no diagnóstico do refluxo..$^{5-7}$ Em relação à avaliação de refluxo gastroesofágico utilizando técnicas de radiografia com contraste, a cintilografia apresenta sensibilidade similar, sendo que alguns trabaIhos mostram uma sensibilidade superior das técnicas radiológi$\operatorname{cas}^{3,7}$, e outros da técnica cintilográfica', porém, a cintilografia possui as vantagens de expor o paciente a doses menores de radiação e de ser mais fisiológical,8.

$\mathrm{Na}$ realização deste exame cintilográfico é habitual que além da pesquisa de refluxo gastroesofágico se adquira imagem tardia dos campos pulmonares cinco horas após a ingestão do radiofármaco. Isto se deve a relatos de que esta imagem tardia é útil na detecção de possíveis episódios de microaspiração do material refluído, principalmente se os episódios de refluxo atingirem o terço superior do esôfago e a boca. Apesar da microaspiração ser responsável pelo quadro clínico pulmonar de alguns dos pacientes, que costumam apresentar tosse e broncospasmo, atualmente acredita-se que os sintomas descritos podem ser decorrentes também de mecanismos de estimulação vagal desencadeados pela irritação da parede do esôfago pelo conteúdo ácido do estômago?. Apesar de alguns trabalhos mostrarem alta taxa de positividade da imagem tardia de campos pulmonares na detecção de aspiraçãa pulmonar ${ }^{10-}$ 12, corroborando a hipótese de que a microaspiração é a responsável por parte do quadro pulmonar associado ao refluxo gastroesofágico, outros trabalhos mostram taxa de positividade limitada ou mesmo nula, sugerindo ser a estimulação vagal a principal responsável pelas queixas pulmonares ${ }^{13-14}$. Devido aos relatos de literatura de que a cintilografia pode detectar episódios de aspiração pulmonar, alguns médicos ainda solicitam, ou esperam que seja realizada, a imagem tardia de campos pulmonares como parte da cintilografia para avaliação de refluxo gastroesofágico. Desta forma, em nosso serviço temos realizado de maneira rotineira a imagem tardia de campos pulmonares cinco horas após a ingestão do radiofármaco, porém sem observarmos resultados positivos nesta etapa do exame.

\section{Métodos}

Foram retrospectivamente analisados todos os laudos de cintilografia para a avaliação de refluxo gastroesofágico realizados de 20/0I/200 I até 28/03/2006. Neste período foram realizados 
755 exames para avaliação de refluxo gastroesofágico, sendo que em 753 foi realizada imagem tardia para avaliação de aspiração pulmonar. Quatrocentos exames foram realizados em pacientes do sexo masculino e 355 em pacientes do sexo feminino, a idade média dos pacientes foi de 5,18 anos, desvio-padrão de $\pm 11,3$ anos, variando de I mês até 94,5 anos. Os exames foram analisados segundo a presença ou ausência de refluxo; sua frequêencia, classificada em seis categorias baseadas no número de episódios: I, 2, 3, 4, 5 e >5 episódios; sua intensidade, classificada em três categorias: discreta, moderada e acentuada; e a altura do esôfago atingida pelo refluxo, classificada em quatro categorias: terço inferior, terço médio, terço superior e boca. Estas classificações foram realizadas de maneira subjetiva pelo médico nuclear no momento da realização do laudo. Avaliou-se também a presença de atividade radioativa em campos pulmonares nas imagens tardias.

O exame de cintilografia de refluxo gastroesofágico foi realizado, como já especificado acima, em duas etapas. Na primeira etapa, imagens na projeção anterior de abdome superior e tórax foram obtidas a cada 15 segundos durante 45 minutos (Figura I) após a ingestão de 6 a $12 \mathrm{MBq}$ de estanho coloidal diluído em aproximadamente $30 \mathrm{ml}$ de líquido (água ou leite), seguido por ingestão de cerca de $200 \mathrm{ml}$ de líquido (água ou leite) para a limpeza da atividade radioativa residual na cavidade oral e esôfago. Na segunda etapa foram obtidas imagens nas projeções anterior e posterior do tórax cerca de quatro a cinco horas após a ingestão do radiofármaco (Figura 2). Durante o intervalo entre as duas etapas o paciente era liberado para desempenhar suas atividades habituais.

\section{Resultados}

Dos 755 exames realizados no período, 307 apresentaram episódios de refluxo gastroesofágico. Em relação à freqüência de episódios de refluxo por exame, em 84 exames observou-se um episódio, 51 apresentaram dois episódios, 45 apresentaram três, 37 apresentaram quatro, 18 apresentaram cinco e em 72 observaramse mais do que cinco episódios. Com relação à intensidade dos episódios de refluxo, 179 exames apresentaram refluxo de grau discreto, 69 de grau moderado, 37 de grau acentuado e em 22 exames o grau de refluxo não foi registrado no laudo. No que tange à altura atingida pelo refluxo, 46 exames apresentaram refluxo até 0 terço inferior do esôfago, 83 até o terço médio, 161 até o terço superior, II até a boca e seis exames não tiveram a altura do refluxo registrada no laudo. A imagem tardia foi realizada em 753 exames, não se observando nenhum episódio de aspiração pulmonar.

\section{Discussão}

A cintilografia para avaliação de refluxo gastroesofágico tem se mostrado útil no diagnóstico e seguimento dos pacientes com esta patologia. Apesar de inicialmente ter se preconizado a realização de imagens tardias de campos pulmonares para a pesquisa de aspiração pulmonar, alguns trabalhos mostram que esta imagem é pouco útil devido a sua baixa positividade ${ }^{13-14}$. Na nossa série de 755 exames, nenhum dos 753 exames em que foi realizada a imagem tardia observou-se positividade nesta etapa. Os motivos desta baixa
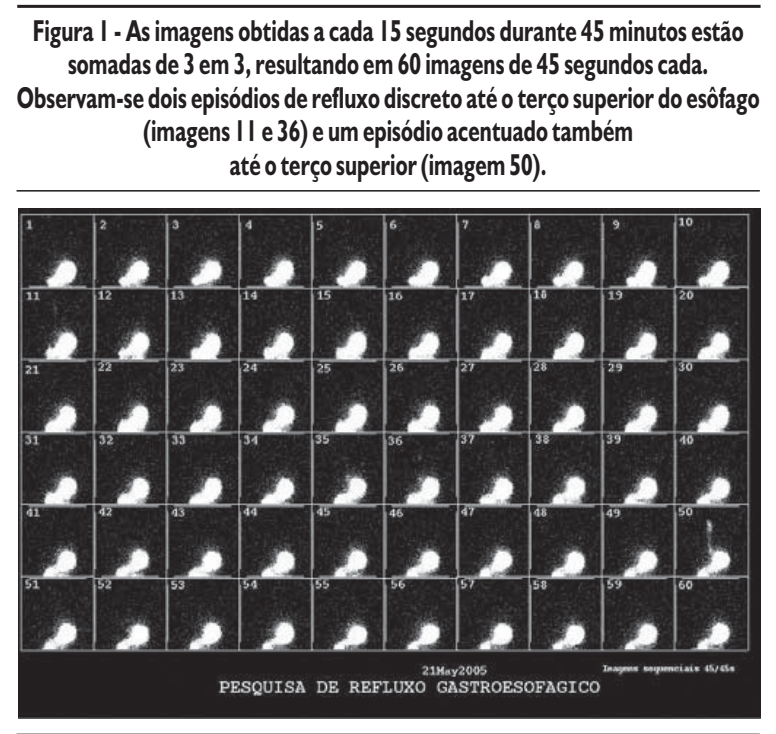

Figura 2 - Imagens anterior e posterior de tórax obtidas 5 horas após a ingestão do radiofármaco. Nota-se ausência de captação em campos pulmonares.

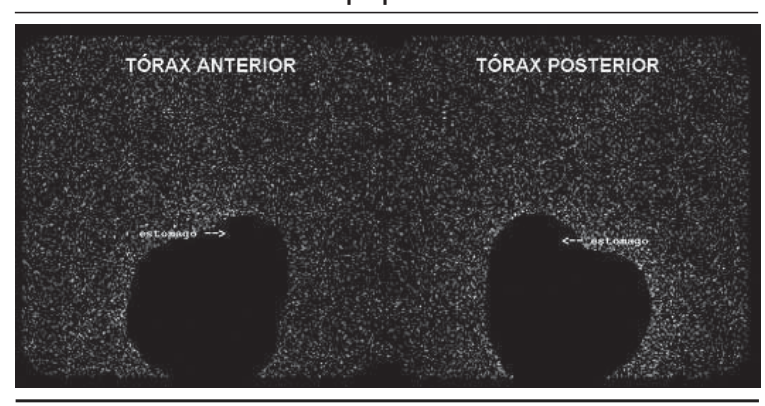

positividade podem ser: a raridade da ocorrência de aspiração pulmonar nestes pacientes, visto que a minoria deles apresenta refluxo até a boca, e mesmo nesta situação, se os mecanismos responsáveis pela deglutição estiverem íntegros, dificilmente 0 material refluído chegará às vias respiratórias. Uma outra causa da baixa positividade do método pode ser a sua sensibilidade limitada para a detecção de microaspiração, principalmente se esta se localizar na base pulmonar, onde será difícil diferenciar a discreta atividade no pulmão da atividade radioativa expressiva em estômago e nas alças intestinais próximas ao diafragma ${ }^{15}$.

Independente do motivo da baixa positividade da imagem tardia realizada de quatro a cinco horas após a administração do material radioativo, esta parece ser pouco útil na investigação rotineira dos pacientes com suspeita de refluxo gastroesofágico e deve, na medida do possível, se restringir a pacientes em que a probabilidade de aspiração seja maior (sintomas pulmonares mais exuberantes e patologias associadas que facilitem a aspiração). Apesar da realiza- 
ção da imagem tardia não significar um incremento da radiação recebida pelo paciente, ela representa um incômodo para este e para os familiares que têm que retornar ao local de realização do exame algumas horas após a primeira etapa, além de encarecer desnecessariamente o procedimento, ao aumentar o tempo de uso do equipamento de detecção das imagens.

Uma importante consideração a ser feita é o motivo de alguns trabalhos mostrarem positividade alta para a detecção de aspiração pulmonar. Quando analisamos alguns destes trabalhos observamos que, além de restringirem a análise a grupos de pacientes com sintomatologia pulmonar evidente, as imagens tardias são realizadas no dia seguinte à ingestão do radiofármaco, após o paciente ter dormido 10,12. Em grupos de pacientes asmáticos, a imagem tardia no dia seguinte à administração do radiofármaco tem mostrado positividade variável (0\% a 75\%) quando diversos trabalhos são avaliados ${ }^{1520}$. Com relação às imagens após o sono (cerca de 10 a 15 horas após a ingestão do radiofármaco), apesar deste não ser o protocolo utilizado para a realização da segunda etapa em nosso serviço, talvez esta seja uma maneira mais adequada para a investigação de aspiração pulmonar do que a realização de imagens cinco horas após a dose. No entanto, a aquisição de imagens tardias apresenta algumas complicações na sua realização, como a necessidade da administração do material radioativo pouco tempo antes do paciente deitar, a fim de que uma parte deste material ainda esteja no estômago durante o sono. Se este material for administrado algumas horas antes do paciente deitar, possivelmente a maior parte dele já se encontrará em alças intestinais, diminuindo a sensibilidade do método.

Para diminuir a velocidade com que o material deixa o estômago, alguns pesquisadores sugerem que se marquem alimentos sólidos com o material radioativo, ou mesmo que administre uma mistura de alimentos sólidos e líquidos marcados. Enquanto os líquidos têm a vantagem de aumentar a sensibilidade para a detecção do refluxo, os sólidos podem ser mais apropriados para a detecção de episódios tardios de aspiração. Crausaz et al. ${ }^{16}$, por exemplo, relatam uma sensibilidade de $75 \%$ para a detecção de aspiração pulmonar nas imagens realizadas na manhã seguinte ao início do exame, quando utilizaram uma mistura de alimentos radioativos sólidos e líquidos. Desta forma, talvez o mais adequado seja realizar a pesquisa de refluxo e a pesquisa de aspiração como dois exames distintos, sendo que na pesquisa de refluxo utilizaríamos líquidos marcados, e na pesquisa de aspiração investigaríamos a presença de atividade radioativa pulmonar na manhã seguinte ao paciente ter recebido o material radioativo marcando alimentos sólidos e deitado logo em seguida.

\section{Conclusão}

A realização de maneira rotineira da imagem tardia dos campos pulmonares quatro a cinco horas após a ingestão do radiofármaco, a fim de investigar a presença de aspiração em pacientes submetidos à cintilografia para avaliação de refluxo gastroesofágico é pouco útil, devendo-se reservar esta etapa do procedimento para aqueles pacientes com maior probabilidade de aspiração.
Conflito de interesse: não há.

\section{SUMMARY}

EVALUATION OF THE USEFULNESS OF ASSESSING PULMONARY ASPIRATION IN A GASTROESOPHAGEAL REFLUX SCINTIGRAPHY STUDY

OBJECTIVE. To evaluate the benefit of routine pulmonary imaging with a five hour delay in gastroesophageal reflux scintigraphy (GERS) to detect pulmonary aspiration.

Methods. 755 cases were analyzed (400 men, 355 women) within a wide range of age (I month up to 94 years, mean of 5.2 years). Presence of reflux, its frequency, severity, and esophageal extension were investigated. Delayed pulmonary images assessed the presence of radioactivity in the lungs.

RESULTS. 307 cases of gastroesophageal reflux were identified, 84 of which with just one episode, 5/ with 2 episodes, 45 with 3, 37 with 4,18 with 5, and 72 with more than 5. Concerning severity of these 307 cases 22 had no evaluation, 179 were mild, 69 intermediate, and 37 severe. Degree of esophageal extension was not recorded for 6 cases; in 46 cases reflux covered the lower third of the esophagus, in 83 cases it reached the central third, in 161 the upper third, and in // cases it extended to the mouth. Delayed pulmonary images were available in 753 cases and none showed signs of lung aspiration.

CONCLUSIONs. Delayed pulmonary images were negative in all cases regardless of the patients' age or severity of the disease. This is suggestive that either lung aspiration is rare in GERS procedures or delayed pulmonary imaging has a sensitivity that is too low to permit detection. [Rev Assoc Med Bras 2007; 53(3): 257-60]

KEY woRDS: Scintigraphym Reflux. Aspiration. Lung. Gastroesophageal. Gastroesophageal reflux.

\section{REFERÊNCIAS}

I. Rudd TG, Christie DL. Demonstration of gastroesophageal reflux in children by radionuclide gastroesophagography. Radiology. 1979;131: 483-6

2. Blumhagen JD, Rudd TG, Christie DL. Gastroesophageal reflux in children: radionuclide gastroesophagography. AJR Am J Roentgenol. 1980: 135:100|-4

3. Balson BM, Kravitz EK, McGeady SJ. Diagnosis and treatment of gastroesophageal reflux in children and adolescents with severe asthma. Ann Allergy Asthma Immunol. 1998:81:159-64.

4. Patwari AK, Bajaj P, Kashyp R, Anand VK, Gangil A, Jain A, et al. Diagnostic modalities for gastroesophageal reflux. Indian J Pediatr. 2002;69:133-6.

5. Tolia V, Calhoun JA, Kuhns LR, Kauffman RE. Lack of correlation between extended $\mathrm{pH}$ monitoring and scintigraphy in the evaluation of infants with gastroesophageal reflux. J Lab Clin Med. 1990; 1 15:55963

6. Vandenplas Y, Derde MP, Piepsz A. Evaluation of reflux episodes during simultaneous esophageal $\mathrm{pH}$ monitoring and gastroesophageal reflux scintigraphy in children. J Pediatr Gastroenterol Nutr. 1992;14:25660

7. El Mouzan MI, Abdullah AM. The diagnosis of gastroesophageal reflux disease in children. Saudi Med I. 2002:23:164-67.

8. Siegel JA, Wu RK, Knight LC, Zelac RE, Stern HS, Malmud LS. Radiation dose estimates for oral agents used in upper gastrointestinal disease. J Nucl Med. 1983;24:835-7. 
Gouvea A et al.

9. Stein MR. Possible mechanisms of influence of esophageal acid on airway hyperresponsiveness. Am J Med. 2003; I I5(Suppl 3A):55S-9S.

1 0. Crausaz F, Bischof-Delaloye A, Cretin T, Delaloye B, Gonvers |J, Favez G. Gastroesophageal reflux and nocturnal broncho-aspiration in chronic bronchopathies. Schweiz Med Wochenschr. 1985; I I 5:994-5.

I I. Veyrac M, Bories P, Collet H, Parelon G, Fauroux P, Godard P, et al. Esophageal scintigraphy and $\mathrm{pH}$ measurement in asthmatic adults suspected of having gastroesophageal reflux. Gastroenterol Clin Biol. 1986:10:400-4

12. Bestetti A, Carola F, Carnevali-Ricci P, Sambataro G, Tarolo GL. $99 \mathrm{mTc}$-sulfur colloid gastroesophageal scintigraphy with late lung imaging to evaluate patients with posterior laryngitis. I Nucl Med, 2000;41: | 597-602.

13. Latini G, Del Vecchio A, De Mitri B, Giannuzzi R, Presta G, Quartull $L$, et al. Scintigraphic evaluation of gastroesophageal reflux in newborns. Pediatr Med Chir, 1999:21:115-7.

14. Le Luyer B, Texte D, Segond G, Mallet E, de Menibus CH. Value and role of esophageal scintigraphy in the diagnosis of gastroesophageal reflux in infants and children. Pediatrie, 1983;38:291-302.

1 5. Ruth M, Carlsson S, Mansson I, Bengtsson U, Sandberg N. Scintigraphic detection of gastro-pulmonary aspiration in patients with respiratory disorders. Clin Physiol. 1993;13:19-33.
16. Crausaz FM, Favez G. Aspiration of solid food particles into lungs of patients with gastroesophageal reflux and chronic bronchial disease. Chest. 1988;93:376-8.

17. Chernow B, Johnson LF, Janowitz WR, Castell DO. Pulmonary aspiration as a consequence of gastroesophageal reflux: a diagnostic approach. Dig Dis Sci. 1979:24:839-44.

18. Ducolone A, Vandevenne A, Jouin H, Grob JC, Coumaros D, Meyer C, et al. Gastroesophageal reflux in patients with asthma and chronic bronchitis. Am Rev Respir Dis. 1987;135:327-32.

19. Ghaed N. Stein MR. Assessment of a technique for scintigraphic monitoring of pulmonary aspiration of gastric contents in asthmatics with gastroesophageal reflux. Ann Allergy. 1979;42:306-8.

20. Reich SB, Earley WC, Ravin TH, Goodman M, Spector S, Stein MR Evaluation of gastro-pulmonary aspiration by a radioactive technique: concise communication. J Nucl Med. 1977; 18:1079-81.

Artigo recebido: 23/1 I/06 Aceito para publicação: 02/03/07 